\title{
Detailed mathematical and numerical analysis of a dynamo model
}

\author{
Aditi Sood and Eun-jin Kim
}

\author{
School of Mathematics and Statistics, University of Sheffield, S3 7RH, UK \\ e-mail: [smp11as;e.kim]@sheffield.ac.uk
}

Received 25 May 2013 / Accepted 31 December 2013

\begin{abstract}
We investigate the role of nonlinear feedback by $\alpha$-quenching, flux losses, and feedback by differential rotations in dynamo. Specifically, by studying the nonlinear dynamo model analytically and numerically, we unfold how frequency $p$ of magnetic field, magnetic field strength $|B|$, and phase $\phi$ are influenced by different types of nonlinear feedback in the limit of a very weak mean and/or fluctuating differential rotation. We find that $p$ and $\phi$ are controlled by both flux losses with no influence by $\alpha$-quenching when there is no back reaction because of fluctuating differential rotation. We find a similar effect of poloidal flux loss and toroidal flux loss on $p$ and $|B|$ in the absence of a back reaction of shear. Their effect becomes totally different with the inclusion of this back reaction. Detailed investigations suggest that toroidal flux loss tends to have more influence than poloidal flux loss (with or without $\alpha$-quenching) in the presence of fluctuating shear. Furthermore, the effect of $\alpha$-quenching is boosted when combined with toroidal flux loss, indicating that the dynamic balance of dynamo is optimized in the presence of both $\alpha$-quenching and flux loss. These results highlight the importance of nonlinear transport coefficients and differential rotation in the regulation of a dynamo.
\end{abstract}

Key words. dynamo - stars: magnetic field - stars: evolution - stars: solar-type - stars: rotation

\section{Introduction}

Over the decades it has been revealed through high-resolution observations that the magnetic field on the solar surface is very structured, consisting of small-scale, rapidly changing magnetic fields, which seem to emerge randomly and are highly variable. At the same time, solar magnetic field shows a remarkable degree of organization on a global scale, displaying the butterfly diagrams and polarity reversal with quite a regular cycle of 22 years. These observations can be understood in terms of the amplification of magnetic field by inductive motion of a conducting fluid in the framework of magnetohydrodynamics (MHD) (e.g., Krause \& Rädler 1980) by using a nonlinear dynamo theory. Specifically, the coexistence of regular and complex behaviour of solar magnetic field can be due to the dynamic balance among various nonlinear interactions. It is thus important to understand the role of nonlinear interaction in the regulation of dynamo.

To understand the nonlinear dynamo process, dynamical systems are the simplest tools because they have the capability of exhibiting complex dynamics as (control) parameters of the system change. A notable study of dynamo was previously done by Weiss et al. (1984) by using dynamical model, who illustrated in detail the transition from regularity to chaos as the control parameter (the so-called dynamo number) varies. In particular, Jones et al. (1985) were able to explain the existence of aperiodic magnetic cycle in a dynamical system given by complex Lorenz system, even without an explicit stochasticity (e.g., in parameters, external forcing, etc.).

Based upon the traditional $\alpha-\Omega$ dynamo (Parker 1955; Moffat 1978; Parker 1979), the dynamo number in this work, which controls the efficiency of dynamo action, is proportional to the product of $\alpha$ and $\Omega$ effects, which generate a toroidal field from a poloidal field by cyclonic convection ( $\alpha$ effect) and a poloidal field from a toroidal field by shearing ( $\Omega$ effect).
There is also a dynamical model (Charbonneau 2005) based on flux transport dynamo (another popular dynamo model), which involves the global transport of the magnetic fields via meridional circulations, as well as buoyant rise in magnetic flux (Dikpati \& Charbonneau 1999; Rempel 2006). Weiss et al. (1984) explained the existence of aperiodic magnetic cycles using a simple parameterized model. Since then, various studies have revealed that with a certain set of conditions, low-order models are able to capture the basic behaviour of solar and stellar magnetic fields (Tobias et al. 1995; Weiss et al. 2001; Mininni et al. 2001; Pontieri et al. 2003; Wilmot-Smith et al. 2005; Passos \& Lopes 2008; Lopes \& Passos 2009, 2011). A continuous effort has been devoted to further developing dynamo models to explain interesting properties of solar magnetic activity, as well as solar differential rotational profile, uncovered by high-resolution observational data (Yoshimura 1975, 1978; Jepps 1975; Ivanova \& Ruzimikin 1977).

To understand the effect of various nonlinear interactions that could be important in the evolution of stellar rotation and magnetic fields over the course of spin-down, we revisit a simple parameterized dynamo model that can serve as a full dynamo model. In fact, the observations of the evolution of magnetic fields and rotation of other stars of different ages with different rotation rates have been providing us with valuable information about the relation among rotation, differential rotation, and magnetic activity, which can be utilized to test a dynamo theory against observations for improvement, which can work not only for the Sun but also for other solar-type stars with different rotation rates. The exploration of the relation between magnetic activity and rotation for a broad range of rotation rates is, however, a very challenging problem and cannot be done practically by full MHD simulations owing to the required computational demand (e.g., the resolution of a broad range of length and time scales, etc.). An illustrative theoretical model for a minimal number of key quantities is thus valuable for gaining 
insight into this problem. This is particulary the case in view of the limitations inherent in any model (e.g., in parameterizations of transport coefficients in MHD simulations). We note that a simple parameterized model has attracted a lot of attention for fusion plasmas as a useful model for understanding regulation and bifurcation (L-H transition), which is crucial for improving plasmas (Zhu et al. 2013; Malkov et al. 2009; Kim \& Diamond 2003).

The main purpose of this paper is to present detailed analysis of nonlinear dynamo models to elucidate the effect/role of various nonlinear interactions that have been proposed in previous works and thus to identify self-regulatory behaviour in a viable solar/stellar dynamo model that can explain observations. To this end, we first examine the nonlinear effect through nonlinear transport coefficients ( $\alpha$-quenching and flux losses) and feedback of differential rotation on frequency $p$ and magnetic field strength $|B|$. Our focus would be the feedback mechanism of the Lorentz force on $\alpha$-effect, differential rotation and the enhanced magnetic dissipation through flux losses. Considering observations, we then demonstrate that regulation by the "near" balance between the (nonlinear) generation and destruction of the magnetic fields is a desirable property of a successful model. In particular, this dynamic balance leads to the almost linear increase in frequency with rotation rate, flattening of magnetic energy for high rotation, and quenching in total shear consistent with observation.

The remainder of this paper is organized as follows. In Sect. 2, we introduce our model. The detailed analysis of this model is provided step by step in Sects. 3 to 5: Sect. 3 discusses nonlinear effects through transport coefficients and Sect. 4 (Sect. 5) focuses on nonlinear effects through mean (fluctuating) differential rotation. We explain the implications of our results, together with key observational data in Sect. 6. Section 7 contains the conclusions.

\section{Model equations}

The nonlinear dynamo model under investigation is the dynamical system consisting of the seven coupled ordinary differential equations (ODE) (Sood \& Kim 2013; Weiss et al. 1984),

$$
\begin{aligned}
& \left(\partial_{t}+F_{2}\right) A=2 D B, \text { where } D=\frac{D_{0}}{F_{1}} \\
& \left(\partial_{t}+F_{3}\right) B=\mathrm{i}\left(1+w_{0}\right) A-\frac{1}{2} \mathrm{i} A^{*} w \\
& \left(\partial_{t}+v_{0}\right) w_{0}=\frac{1}{2} \mathrm{i}\left(A^{*} B-A B^{*}\right) \\
& \left(\partial_{t}+v\right) w=-\mathrm{i} A B
\end{aligned}
$$

which are given in a dimensionless form. Here, $A$ is the poloidal magnetic flux, $B$ is the toroidal magnetic field, $w_{0}$ and $w$ are mean and fluctuating differential rotations with zero and twice the frequency of $A$ and $B$, respectively, which are generated by a nonlinear back reaction of $A$ and $B$. Poloidal magnetic field $A$, toroidal magnetic field $B$, and fluctuating differential rotation $w$ are complex, whereas mean differential rotation $w_{0}$ is real. The complex conjugate of $A$ is represented by $A^{*}$. We note that in these dimensionless units, the total mean differential rotation is given by $1+w_{\circ}=\frac{\Delta \Omega}{\Omega}$; thus, mean differential rotation $w_{0}$, due to the back reaction, appears with a negative sign to reduce the total shear from 1 to $1+w_{0}<1$. This is because the differential rotation is inhibited by the tension in the magnetic field lines via Lorentz force (i.e., causing the quenching of $\Omega$-effect.).
Viscosity of mean and fluctuating differential rotations are represented by $v$ and $v_{0}$, respectively, which are considered to be constant.

The parameter $D$ in Eq. (1) involves the dynamo number $D_{0} \propto \alpha \Omega$, which is the key (dimensionless) control parameter. And $F_{1}, F_{2}$, and $F_{3}$ are "nonlinear" transport coefficients, defined as

$$
\begin{aligned}
& F_{1}=1+\kappa_{1}|B|^{2}, \\
& F_{2}=1+\lambda_{1}|B|^{2}, \\
& F_{3}=1+\lambda_{2}|B|^{2},
\end{aligned}
$$

where $\kappa_{1}, \lambda_{1}$, and $\lambda_{2}$ are constant parameters; $F_{1}$ represents the $\alpha$ source term (i.e., helicity by the magnetic field) due to the back reaction by magnetic field that was studied in previous works including Pouquet et al. (1976) and Kleeorin et al. (1982). The enhanced magnetic dissipation through the loss of toroidal and poloidal magnetic fluxes is represented by $F_{2}$ and $F_{3}$, respectively, which removes magnetic flux from the region in which the dynamo operates. We note that this is totally different from the assumption of reduction in magnetic dissipation used in some previous works. In this model, we assume that $\alpha$ is a parameter that increases with rotation as $\alpha \propto \Omega$ based on an $\alpha \Omega$ dynamo, but we envision that $\alpha$ captures other physical mechanisms that increase linearly with $\Omega$. Therefore, dynamo number $D_{0}$ is scaled with rotation rate $\Omega$ as $D_{0} \propto \Omega^{2}$ (Sood \& Kim 2013). The proportionality constant between $D_{0}$ and $\Omega^{2}$ is taken to be unity in the paper for simplicity. In this paper, our model focuses on the dynamic balance through $F_{1}-F_{3}$. Other possible feedback, such as the Malkus-Proctor effect (1975) or $\Lambda$-quenching (Kitchatinov et al. 1994), will be left for future work. It is important to note that according to our non-dimensionlization, physical poloidal magnetic field $A$ is obtained by dividing our dimensionless poloidal magnetic flux $A$ in Eqs. (1)-(4) by rotation $\Omega$.

Expecting the regulation in a nonlinear dynamo model, which can weaken (suppress) the chaotic solution, we make an ansatz for the finite-amplitude solution to Eqs. (1)-(4) in the following system

$A=a \mathrm{e}^{\mathrm{i} p t}, B=b \mathrm{e}^{\mathrm{i}(p t+\phi)}, w=c a^{2} \mathrm{e}^{2 \mathrm{i} p t}$,

where $a>0$ and $b>0$ are assumed to be positive real; $c$ is a complex constant; $p$ is real angular frequency; $\phi$ is the phase difference between toroidal $b$ and poloidal $a$ magnetic fields. Using finite amplitude solution of these forms in Eqs. (1)-(4) gives us the following five relations among $a, b, \phi, p$, and $w_{0}$

$$
\begin{aligned}
& -p^{2}+F_{2} F_{3}=\frac{-\left(2 p^{2}+F_{2} v\right) \epsilon a^{2}}{2\left(4 p^{2}+v^{2}\right)} \\
& \left(F_{2}+F_{3}\right) p=2 D-\frac{p a^{2}}{v_{0}}+\frac{\left(2 F_{2}-v\right) p \epsilon a^{2}}{2\left(4 p^{2}+v^{2}\right)}
\end{aligned}
$$

$w_{0}=\frac{-p a^{2}}{2 v_{0} D}$

$a=\frac{2 D b}{\sqrt{p^{2}+F_{2}^{2}}}$,

$\tan \phi=\frac{p}{F_{2}}$,

where $D=\frac{D_{0}}{F_{1}}, F_{1}=1+\kappa_{1} b^{2}$, and $F_{2,3}=1+\lambda_{1,2} b^{2}$. In the above equations, $\epsilon$ is the parameter tracking the presence of fluctuating shear $w$ as

$\epsilon= \begin{cases}1, & w \neq 0 \\ 0, & w=0\end{cases}$ 
Therefore, when there is no fluctuating shear $(\epsilon=0)$, Eqs. (6), (7) are simplified as

$$
\begin{aligned}
& p= \pm \sqrt{F_{2} F_{3}}, \\
& \left\{\left(F_{2}+F_{3}\right)+\frac{4 D_{0}^{2} b^{2}}{v_{0} F_{1}^{2} F_{2}^{2}\left(F_{2}+F_{3}\right)}\right\} F_{1} \sqrt{F_{2} F_{3}}=2 D_{0},
\end{aligned}
$$

where Eqs. (11) and (9) were used in obtaining Eq. (12). It is worth noting that neglecting nonlinear terms in the above equations (i.e., $F_{1}=F_{2}=F_{3}=1, w_{0}=0$ ) gives the linear dispersion relation for the onset of bifurcation as $p=1$ and $\phi=\pi / 4$.

Interestingly, Eq. (11) shows that the frequency $p$ of the magnetic field is determined by the dissipation of both the magnetic fields, given by the geometric mean of $F_{2}$ and $F_{3}$. Thus $p$ can increase with $b$ due to the enhanced magnetic dissipation given by $F_{2}=1+\lambda_{1} b^{2}$ or $F_{3}=1+\lambda_{2} b^{2}$. In contrast, $p$ is not influenced by $\alpha$-quenching $\left(F_{1}\right)$, demonstrating different effects on the frequency $p$ of $\alpha$-quenching $\left(F_{1}\right)$ and of flux losses $\left(F_{2,3}\right)$. The flux losses $F_{2}$ and $F_{3}$ determine the phase $\phi$ between $b$ and $a$ as $\phi=\sqrt{F_{3} / F_{2}}$; that is, when the toroidal flux loss $F_{3}$ is stronger (weaker) than poloidal flux loss $F_{2}$, it removes $b$ more (less) efficiently than $a$, increasing (decreasing) the phase $\phi$ between $b$ and $a . w_{0}$ in Eq. (8) clearly shows that the sign of $w_{0}$ is negative definite, always reducing the total mean differential rotation $1+w_{0}<1$. Explicit examples of these behaviours are presented in the following Sects. 4,5 through the detailed analysis of finite amplitude solution by taking the different limits of $F_{1}, F_{2}$, and $F_{3}$ as well as $w_{0}, w \rightarrow 0$. This will elucidate the role of $\alpha$-quenching, flux losses, mean and fluctuating differential rotation in self-regulatory behaviour of magnetic activity/cycle. Analytical predictions in Sects. 3-4 are then accompanied by the confirmation by numerical simulations. Specifically, for the numerical parts of our paper, we solve Eqs. (1)-(4) by timestepping for all complex variable $A, B, w$, and $w_{0}$ using $v=0.5$, $v_{0}=35.0$ for different values of $D_{0}$ between 1-400 (See Sood $\&$ Kim 2013 for more details). We also note that for the numerical simulations, the values of the parameters $\kappa_{1}, \lambda_{1}$, and $\lambda_{2}$ are assumed to have the same value, $\kappa_{1}=\lambda_{1}=\lambda_{2} \equiv \lambda=2.5$.

\section{Nonlinear effects through transport coefficients}

The $\alpha$-effect and flux losses are the crucial transport coefficients, capturing the overall effects of small (unresolved) turbulence. The feedback of growing magnetic fields alters the value of these transport coefficients, making them dependent on magnetic fields. These nonlinear transport coefficients provide a way for a dynamo to self regulate. To understand the self regulation through these transport coefficients, it is imperative to investigate the case in the limit of a very weak mean or fluctuating differential rotation by assuming $w_{0}, w \rightarrow 0$. The relevant Eqs. (8)-(12) are then

$$
\begin{aligned}
& p= \pm \sqrt{F_{2} F_{3}}, \\
& \left(F_{2}+F_{3}\right) F_{1} \sqrt{F_{2} F_{3}}=2 D_{0}, \\
& a=\frac{2 D_{0} b}{F_{1} \sqrt{p^{2}+F_{2}^{2}}}, \\
& \tan \phi=\frac{p}{F_{2}} .
\end{aligned}
$$

In the above system, we consider different cases of $F_{1}, F_{2}$, and $F_{3}$ and provide the scalings of $p, b$, and $a$ with rotation.
In the remainder of the paper, the scaling of poloidal field with $\Omega$ in physical units is obtained by dividing $A$ by $\Omega$ because of our nondimensionlization as noted in Sect. 2. Also note that here $a=|A|$ and $b=|B|$.

1. $F_{1}=F=1+\lambda b^{2}, F_{2}=F_{3}=1$. In the presence of $\alpha$-quenching only with no flux loss, Eqs. (13)-(16) are simplified as

$p=1$,

$2\left(1+\lambda b^{2}\right)=2 D_{0}$,

$a=\frac{2 D_{0} b}{\left(1+\lambda b^{2}\right)}$

$\tan \phi=1$.

Equation (17) explicitly shows that while $\alpha$-quenching $\left(F_{1}\right)$ provides nonlinear damping to saturate the growth of magnetic field, it does not influence the frequency $p=1$, which is the value of the frequency at the onset of bifurcation to a finite amplitude solution. Moreover, phase $\phi$ is not altered by $\alpha$-quenching, maintaining a linear value $\frac{\pi}{4}$. In the limit of $D_{0} \gg 1$, Eqs. (18)-(20) enable us to find the scalings of $a$ as $a \sim \Omega$ and and $b$ as $b \sim \Omega$.

We now compare these analytical results by numerical simulation of Eqs. (1)-(4). As noted in Sect. 2, we solve these equations by time stepping and obtain the frequency spectrum by taking a FFT (fast Fourier transform) of the time series of dynamical variable $B$. Once we have the power spectrum we assign colours according to the colour bar next to the figure. We find that analytical scalings are in perfect agreement with the results obtained from numerical simulations shown in Fig. 1 where $\kappa_{1}=\lambda=2.5$ is used. Specifically, Fig. 1a represents the intensity of frequency spectrum for different values of $\Omega$ where the high-to-low intensity is coded in yellow to black colours. A strip of a bright yellow colour tracks the frequency of maximum intensity, which hardly changes with $\Omega$ with the same scaling $(p=1)$ as predicted above. We note that the frequency shown in Fig. 1a is lower than the angular frequency $p$ by a factor of $2 \pi$. Figures $1 \mathrm{~b}-1 \mathrm{c}$ show $b$ and $a$ as functions of $\Omega$, where the scalings similar to the analytic values can be obtained. These results confirm the validity of our finite amplitude solutions. We note that $a \sim \Omega$ implies that the scaling of poloidal magnetic field in the physical unit is $\sim \Omega^{0}$.

2. $F_{1}=1, F_{2}=F_{3}=F=1+\lambda b^{2}$. For simplicity, when poloidal flux loss and toroidal flux loss are enhanced to the same degree while there is no $\alpha$-quenching, Eqs. (13)-(16) become $p \sim\left(1+\lambda b^{2}\right), 2 D_{0}=2\left(1+\lambda b^{2}\right)^{2}, a \sim 2 D_{0} / 2^{1 / 2}\left(1+\lambda b^{2}\right)^{3 / 2}$, and $\tan \phi=1$. Therefore, both flux losses lead to the increase in frequency $p \sim\left(1+\lambda_{1,2} b^{2}\right)$ with $b$, while their effects are canceled in the phase shift $\phi=\frac{\pi}{4}$. The increase of $p$ with $b$ is determined consistently by the nonlinear dynamics, which sets the values of $b$. This dynamical determination of $p$ is reminiscent of the flux transport dynamo where $p$ depends on meridional flow, etc. In the limit of $D_{0} \gg 1$, the scalings of $p, b$, and $a$ are found to be $p \sim \Omega, b \sim \Omega^{\frac{1}{2}}$, and $a \sim \Omega^{\frac{3}{2}}$, which are again confirmed by numerical simulations.

3. $F_{1}=F_{2}=F_{3}=F=1+\lambda b^{2}$. In this case $\alpha$-quenching and flux loss due to poloidal magnetic field and toroidal magnetic field are the same, equally contributing to the flattening of magnetic fields for large rotation, Eqs. (13)-(16) give $p \sim F \sim 1+\lambda b^{2}, D_{0}=\left(1+\lambda b^{2}\right)^{3}, a=2 D_{0} b / 2^{1 / 2}\left(1+\lambda b^{2}\right)^{3}$, and $\tan \phi=1$. Clearly, poloidal flux loss and toroidal flux loss both determine the maximum frequency $p$ of the magnetic field; $p$ increases with $\Omega$, similar to the case where the effect of both 


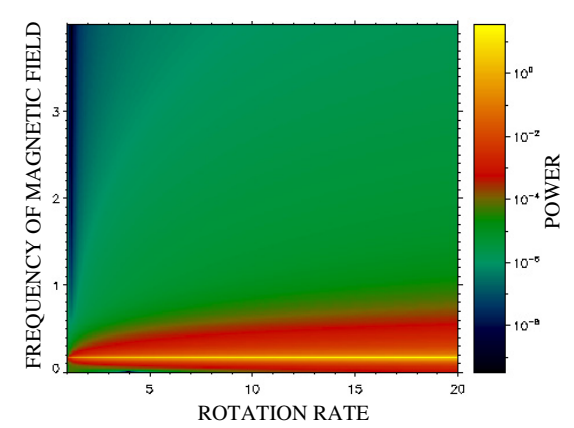

(a) $p$ as a function of $\Omega$

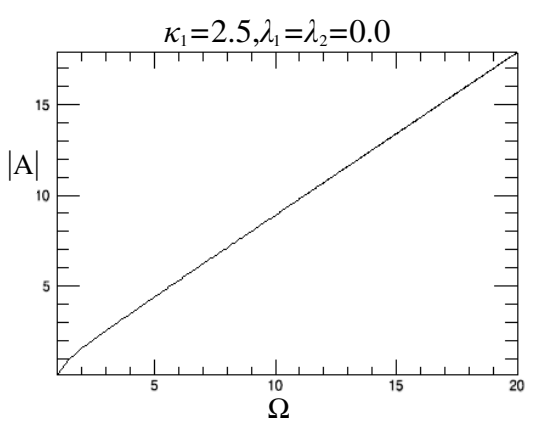

(b) $|A|$ as a function of $\Omega$

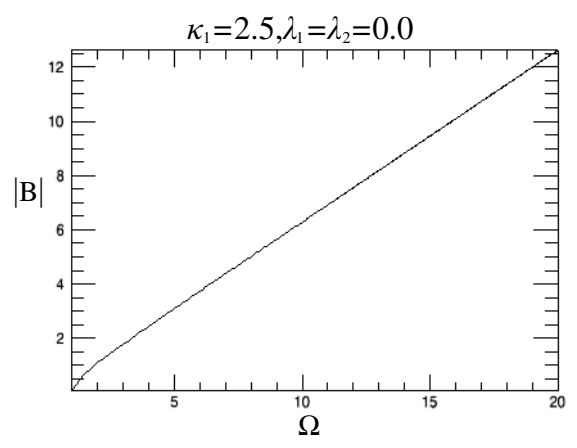

(c) $|B|$ as a function of $\Omega$

Fig. 1. Frequency of maximum intensity $p$, poloidal magnetic field $|A|$ and toroidal magnetic field $|B|$ as a function of rotation rate $\Omega$ for $F_{1}=$ $F, F_{2}=F_{3}=1.0$ for Case 1 in the fourth-order system.

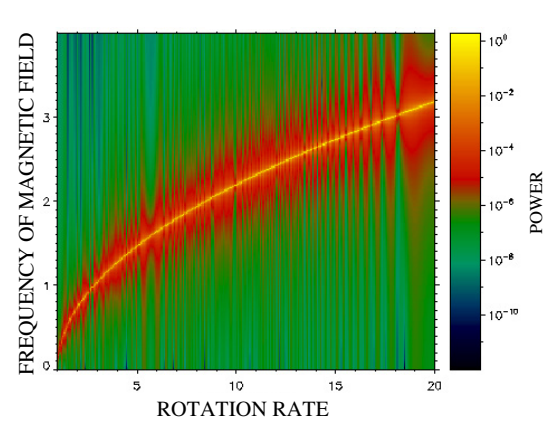

(a) $p$ as a function of $\Omega$

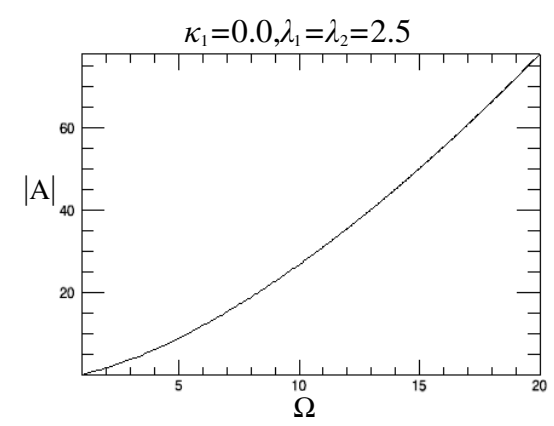

(b) $|A|$ as a function of $\Omega$

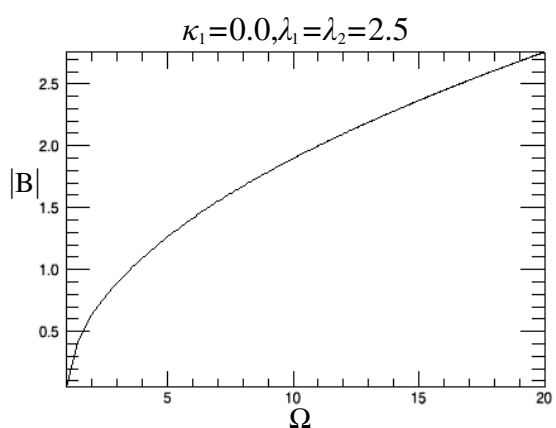

(c) $|B|$ as a function of $\Omega$

Fig. 2. Frequency of maximum intensity $p$, poloidal magnetic field $|A|$ and toroidal magnetic field $|B|$ as a function of rotation rate $\Omega$ for $F_{1}=$ $1.0, F_{2}=F_{3}=F$ for Case 2 in the fourth-order system.

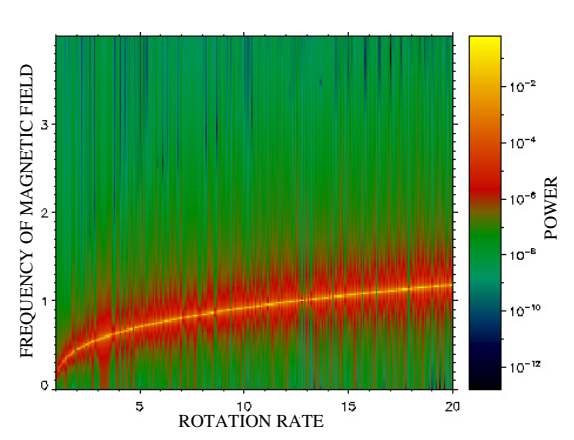

(a) $p$ as a function of $\Omega$

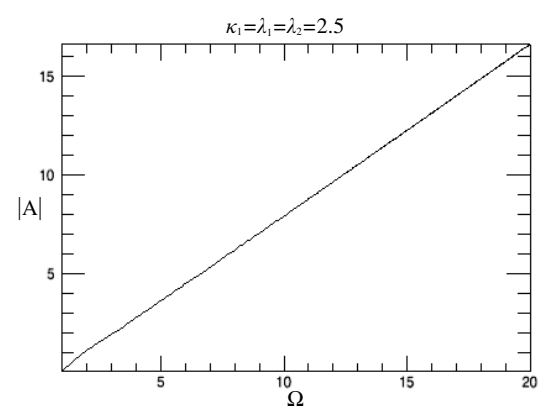

(b) $|A|$ as a function of $\Omega$

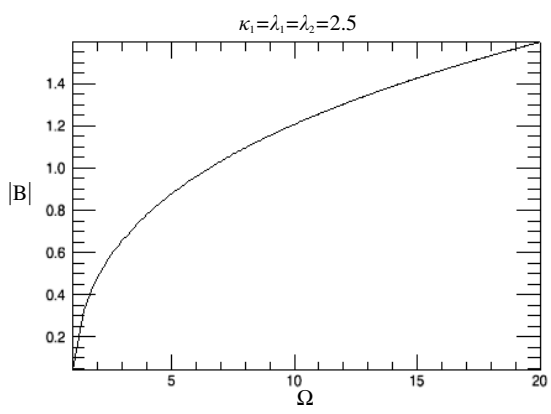

(c) $|B|$ as a function of $\Omega$

Fig. 3. Frequency of maximum intensity $p$, poloidal magnetic field $|A|$ and toroidal magnetic field $|B|$ as a function of rotation rate $\Omega$ for $F_{1}=F_{2}=$ $F_{3}=F$ for Case 3 in the fourth-order system.

flux losses is considered; $a$ and $b$ both increase with rotation rate (cf. Fig. 3a to 3c). Interestingly, the phase between $a$ and $b$ remains unaltered due to the equal amount of $F_{2}$ and $F_{3}$, with its value $\phi=\frac{\pi}{4}$. In the limit of $D_{0} \gg 1$, we find that $p \sim \Omega^{\frac{2}{3}}$, $a \sim \Omega$, and $b \sim \Omega^{\frac{1}{3}}$. Numerically found scalings are almost similar for $p, b$, and $a$ (cf. Table 1). Thus, equal amount of $\alpha$-quenching, enhanced poloidal flux loss and toroidal flux loss lead to stronger reduction in magnetic field for a higher rotation rate, which can provide strong dynamic balance in a nonlinear dynamo.

4. $F_{1}=F_{2}=F=1+\lambda b^{2}, F_{3}=1$. In the presence of only $\alpha$ quenching and enhanced poloidal magnetic flux loss, the various scalings are simplified as $p \sim\left(1+\lambda b^{2}\right)^{1 / 2}, 2 D_{0}=\left(2+\lambda b^{2}\right)^{2}$ $\left(1+\lambda b^{2}\right)^{1 / 2}, a=2 D_{0} b /\left(2+\lambda b^{2}\right)^{1 / 2}\left(1+\lambda b^{2}\right)^{2}, \tan \phi \sim 1 /(1+$ $\left.\lambda b^{2}\right)^{1 / 2}$. Thus, $\phi$ decreases for large $b$ (or large $D_{0}$ ), reducing phase shift between $b$ and $a$ to zero. Furthermore, in the limit of $D_{0} \gg 1$, these give us $p \sim \Omega^{\frac{2}{5}}, b \sim \Omega^{\frac{2}{5}}$, and $a \sim \Omega^{\frac{4}{5}}$.

5. $F_{1}=F_{3}=F=1+\lambda b^{2}, F_{2}=1$. This is the case where $\alpha$-quenching and flux loss due to toroidal magnetic field are in action; Eqs. (13) to (16) give $p \sim\left(1+\lambda b^{2}\right)^{1 / 2}, 2 D_{0}=(1+$ $\left.\lambda b^{2}\right)^{5 / 2}, a=2 D_{0} b /\left(2+\lambda b^{2}\right)^{1 / 2}\left(1+\lambda b^{2}\right)^{2}$, and $\tan \phi=1$. Clearly, frequency $p$ of magnetic field is influenced by toroidal magnetic field $b$. For $D_{0} \gg 1$, the scalings of $p, b$, and $a$ are found to be $p \sim \Omega^{\frac{2}{5}}, b \sim \Omega^{\frac{2}{5}}$, and $a \sim \Omega^{\frac{6}{5}}$. Compared to the Case 4 above, we see that change from toroidal to poloidal flux loss only affects the scaling of $a$ with $\Omega$. 
Table 1. Scaling exponents for $p,|B|$, and $|A|$ for $\Omega \geq 5$ in the cases of the fourth-order system where $p=\Omega^{\xi},|B|=\Omega^{\beta}$, and $|A|=\Omega^{\gamma}$.

\begin{tabular}{lccc}
\hline \hline Transport coefficients & $\xi$ & $\beta$ & $\gamma$ \\
\hline$F_{1}=F, F_{2}=F_{3}=1$ & 0 & 1.0 & 1.0 \\
$F_{1}=1, F_{2}=F_{3}=F$ & 1.0 & 0.50 & 1.55 \\
$F_{1}=F_{2}=F_{3}=F$ & 0.66 & 0.41 & 1.08 \\
$F_{1}=F_{2}=F, F_{3}=1$ & 0.41 & 0.47 & 0.84 \\
$F_{1}=F_{3}=F, F_{2}=1$ & 0.41 & 0.47 & 1.55 \\
$F_{1}=F_{2}=1, F_{3}=F$ & 0.67 & 0.69 & 2.03 \\
$F_{1}=F_{3}=1, F_{2}=F$ & 0.67 & 0.69 & 1.35 \\
\hline
\end{tabular}

6. $F_{3}=F=1+\lambda b^{2}, F_{1}=F_{2}=1$. Here flux loss due to toroidal magnetic field tames the amplitude of $b$ for large $\Omega$, turning Eqs. (13)-(16) into the form $p=\left(1+\lambda b^{2}\right)^{1 / 2}$, $2 D_{0}=\left(2+\lambda b^{2}\right)\left(1+\lambda b^{2}\right)^{1 / 2}, a=2 D_{0} b /\left(1+\lambda b^{2}\right)^{1 / 2}$, and $\tan \phi \sim\left(1+\lambda b^{2}\right)^{1 / 2}$. Therefore, toroidal flux loss increases the frequency according to the strength of $b$, which is determined dynamically. Also, the phase $\phi$ between $a$ and $b$ increases, approaching $\frac{\pi}{2}$ as $D_{0} \gg 1$, confirming that the preferential removal of toroidal flux over poloidal flux increases the phase. In the limit of $D_{0} \gg 1$, we find power-law scalings $p \sim \Omega^{\frac{2}{3}}, b \sim \Omega^{\frac{2}{3}}$, and $a \sim \Omega^{2}$.

7. $F_{2}=F=1+\lambda b^{2}, F_{1}=F_{3}=1$. Flux loss due to poloidal field determines the frequency, simplifying Eqs. (13)-(16) as $p=\left(1+\lambda b^{2}\right)^{1 / 2}, 2 D_{0}=\left(2+\lambda b^{2}\right)\left(1+\lambda b^{2}\right)^{1 / 2}, a=2 D_{0} b /(1+$ $\left.\lambda b^{2}\right)\left(2+\lambda b^{2}\right)^{1 / 2}, \tan \phi=\left(1+\lambda b^{2}\right)^{-1 / 2}$. In the limit of $D_{0} \gg 1$, the scalings are found as $p \sim \Omega^{2 / 3}, b \sim \Omega^{2 / 3}$ and $a \sim \Omega^{4 / 3}$, $\tan \phi \sim \Omega^{-2 / 3}$. Therefore, compared to Case 6 above, the difference is seen in the scalings of $a$ and $\phi$; that is, reduction in the scaling of $a$ and $\phi$ with $\Omega$ is observed, contrary to the case where only saturation mechanism is flux loss due to toroidal magnetic field.

To summarize, the effects of $\alpha$-quenching $F_{1}$ and flux losses $F_{2}$ and $F_{3}$ are shown to be different; $p$ is dynamically determined by $b$ through flux loss $F_{2}$ and $F_{3}$, but with no influence of $\alpha$-quenching $F_{1}$; that is, $p=1$ with $\alpha$-quenching, while $p$ increases with $\Omega$ due to flux loss. The effects of $F_{2}$ and $F_{3}$ are almost similar, leading to the same scalings of $p$ and $b$ with $\Omega$. The phase $\phi$ is determined by the ratio of $F_{2}$ and $F_{3}$. In all cases, $b$ is always found to increase with $\Omega$ as $b \propto \Omega^{\beta}$ where $\beta$ takes the value between $1 / 3$ and 1 . The largest $\beta=1$ is found in the case of $\alpha$-quenching only, while smallest $\beta=0.4$ in the presence of nonlinear terms of same magnitude. Thus, in this fourthorder system, $b$ keeps growing with $\Omega$, without flattening for large $\Omega$. Numerical values of scaling exponents are summarized in Table 1 .

\section{Nonlinear effect through mean differential rotation}

We now incorporate the nonlinear effect due to the modification of differential rotation by magnetic fields and examine the role of mean differential rotation in self-regulatory behaviour. Here, we exclude the fluctuating differential rotation in order to identify the effect of mean differential rotation. The effect of fluctuating differential rotation is presented in Sect. 5. The main equations are Eqs. (6) $-(10)$ for $w=0$, and $v_{0}=35$ is chosen for numerical simulations.

1. $F_{1}=F_{2}=F_{3}=1$. Even in the absence of $\alpha$-quenching or any flux loss, the back reaction of mean differential rotation $w_{0}$ provides the saturation mechanism to ensure a finite-amplitude solution, reducing Eqs. (6)-(10) to

$p= \pm 1$,

$b=D_{0}^{-1 / 2} v_{0}^{1 / 2}\left(1-D_{0}^{-1}\right)^{1 / 2}$,

$a=D_{0}^{1 / 2} v_{0}^{1 / 2} \sqrt{\left(2\left(1-D_{0}^{-1}\right)\right)}$,

$w_{0}=-1+\frac{1}{D_{0}}$,

$\tan \phi=1$.

Equation (21) immediately shows that the frequency of magnetic field is independent of rotation rate, with no influence of $w_{0}$. In the limit of a high rotation rate, i.e. $D_{0} \gg 1$, Eqs. (22)-(25) give us analytical scalings $b \sim \Omega^{-1}, a \sim \Omega$ and total shear $1+w_{0} \sim \Omega^{-2} \ll 1$ for $\Omega \gg 1$. The phase angle between poloidal magnetic field $a$ and $b$ keeps its linear value of $\phi=\frac{\pi}{4}$ (cf. Eq. (25)). We note that the dependence of the total shear on rotation rate $\Omega^{-2}$ shows significant shear quenching. These results are confirmed by numerical simulations. Figure 4 a shows one single dominant frequency, $p \sim 1$, that hardly changes with rotation. A red broad band of weak intensity around the localized frequency of maximum intensity increases its width initially and then decreases for higher rotation. This behaviour arises be cause shear quenching due to Lorentz force is too strong, leading to significant quenching of magnetic field strength $b$ for $\Omega \gg 1$. This severe shear quenching is shown in Fig. $4 d$, where total shear decreases rapidly and approaches zero for a higher rotation rate $(\Omega \geq 12)$. Numerically, scalings of $|B|,|A|$ and total shear $1+w_{0}$ for high rotation are found to be $a \sim \Omega^{1.02}, b \sim \Omega^{-0.98}$, $1+w_{0} \sim \Omega^{-2.0} \sim D_{0}^{-1}$, which are similar to analytically predicted values, noted above.

2. $F_{1}=F=1+\lambda b^{2}, F_{2}=F_{3}=1$. In the presence of $\alpha$-quenching alone with no flux loss, Eqs. (6) to (10) become

$p= \pm 1$,

$F^{2}+\frac{D_{0} b^{2}}{v_{0}}=D_{0} F$

$a=\frac{\sqrt{2} D_{0} b}{F}$,

$w_{0}=-\frac{D_{0} b^{2}}{F v_{0}}$

$\tan \phi=1$

Again, the frequency $p$ is independent of rotation rate. Analytical scalings are obtained from Eqs. (26)-(30) for the limit of the high rotation rate. In the limit of the high rotation rate, $D_{0} \gg 1$, we obtain $b \sim \Omega^{-1}, a \sim \Omega$, and $\left(1+w_{0}\right) \sim \Omega^{-2}$. The total shear $1+w_{0} \sim \Omega^{-2} \ll 1$ for large $\Omega$ implies that Lorentz force generates strong mean differential rotation $w_{0}<0$, causing the significant reduction in the magnetic field.

3. $F_{2}=F_{3}=F=1+\lambda b^{2}, F_{1}=1$. Equations (6) to (10) give us
$p= \pm F$,
$F^{4}+\frac{D_{0} b^{2}}{v_{0}}=D_{0} F^{2}$
$a=\frac{\sqrt{2} D_{0} b}{F^{3 / 2}}$
$w_{0}=-\frac{D_{0} b^{2}}{F^{2} v_{0}}$,
$\tan \phi=1$. 


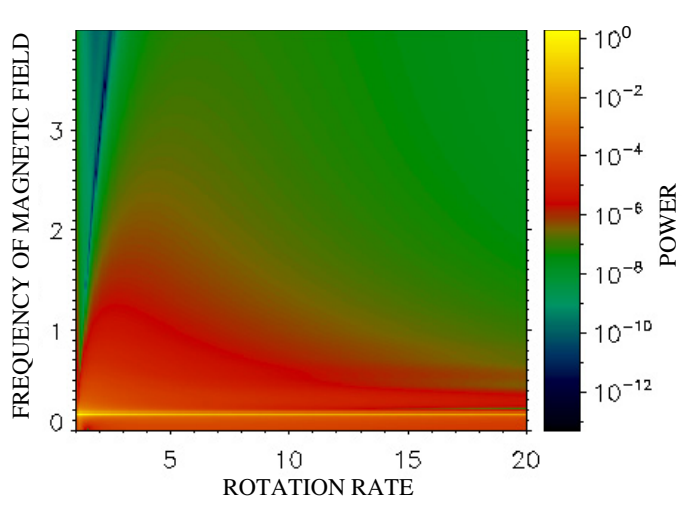

(a) $p$ as a function of rotation rate.

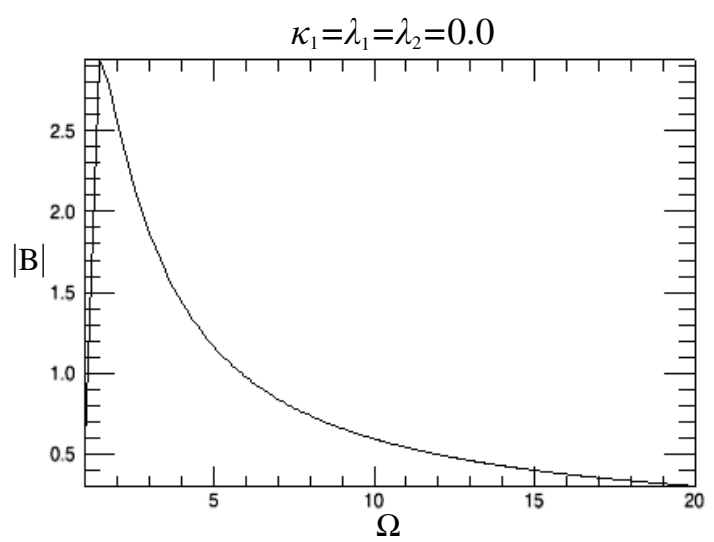

(c) $|B|$ a function of $\Omega$

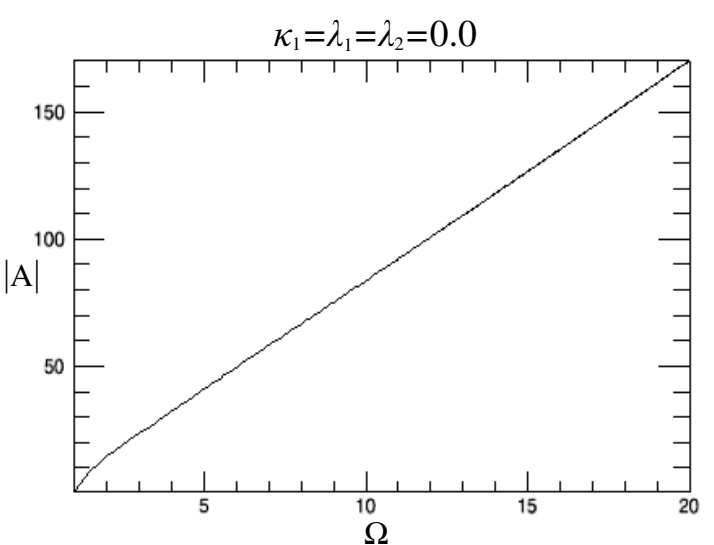

(b) $\mid A$ as a function of $\Omega$

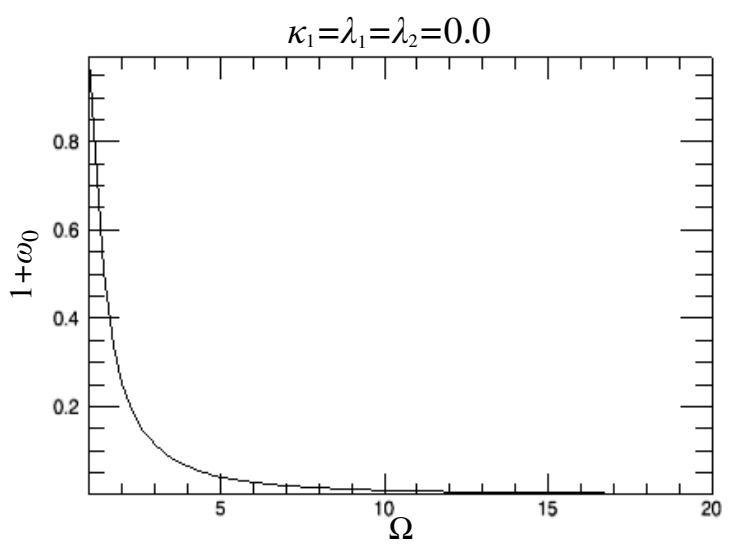

(d) Total shear as a function of rotation rate.

Fig. 4. Frequency of maximum intensity $p$, poloidal magnetic field $|A|$, toroidal magnetic field $|B|$ and total shear as a function of rotation rate $\Omega$ for $F_{1}=F_{2}=F_{3}=1$ for Case 1 in the fifth-order system.

Thus, $p$ changes as $b$ changes with $\Omega$ (cf. Eq. (31)) due to nonlinear flux losses $F_{2}$ and $F_{3}$. In the limit of the high rotation rate, $D_{0} \gg 1$, we find $b \sim \Omega^{-1} \ll 1,1+\lambda b^{2} \sim 1, a \sim \Omega$, and $1+w_{0} \sim \Omega^{-2}$. The decline in toroidal magnetic field with $\Omega$ is again due to the severe shear quenching present in the system. On the other hand, the phase shift between $a$ and $b$ remains as $\frac{\pi}{4}$ owing to equal amount of the toroidal and poloidal magnetic fluxes.

4. $F_{1}=F_{2}=F_{3}=F=1+\lambda b^{2}$. Equations (6)-(10) are simplified as

$p= \pm F$,

$F^{6}+\frac{D_{0} b^{2}}{v_{0}}=D_{0} F^{3}$

$a=\frac{\sqrt{2} D_{0} b}{F^{3 / 2}}$,

$w_{0}=-\frac{D_{0} b^{2}}{F^{2} v_{0}}$,

$\tan \phi=1$.

In this case we numerically find that the presence of $F_{1}, F_{2}$, and $F_{3}$ leads to interesting dynamics for large $\Omega$. For instance, the system bifurcates at $\Omega=19.7$ with a sudden change in $p$, $|B|,|A|$, and total shear (cf. Figs. 5a-5d). This result suggests a possibly dramatic change in the dynamo owing to a strong mean shear. Its physical relevance in the evolution of solar spin-down will be addressed in future work.
5. $F_{1}=F_{2}=F=1+\lambda b^{2}, F_{3}=1$. The presence of $\alpha$-quenching and nonlinear poloidal flux loss reduces Eqs. (6)-(10) to

$p= \pm \sqrt{F}$

$b^{2}=2^{-1} D_{0}^{-1} F^{6}\left(1+F^{-1}\right)\left(F^{1 / 2}-1-F^{-1}\right)$,

$a=\frac{2 D_{0} b}{F^{2} \sqrt{F+1}}$,

$w_{0}=\frac{-p a^{2} F}{2 v_{0} D_{0}}$

$\tan \phi=F^{-1 / 2}$.

We note that $p$ is influenced by $b$ as $p \sim b$ (cf. Eq. (41)). Again, analytical scalings are obtained within the limit of a very high rotation rate, i.e., $D_{0} \gg 1$ as $p \sim \Omega^{2 / 5}, b \sim \Omega^{2 / 5}, a \sim \Omega^{4 / 5}$, and $\left(1+w_{0}\right) \sim \Omega^{-2}$, whereas the phase shift between $a$ and $b$ decreases as rotation rate increases. The analytical scalings for $p$, $|B|$, and $|A|$ are the same as for those obtained in the fourth-order system in the case of $\alpha$-quenching and flux loss. Numerically found scalings are very similar to these analytical results.

Somewhat similar results are found for different cases, as briefly summarized as follows. For $F_{1}=F_{3}=F, F_{2}=1$, and $F_{3}=F, F_{1}=F_{2}=1$, we find that frequency $p$ and phase shift are influenced by $b$. For $F_{2}=F, F_{1}=F_{3}=1, p$ and phase shift are the same as in Case 5. All the numerical results for high rotation, $\Omega \geq 5$ are listed in Table 2 . 
A. Sood and E. Kim: Detailed mathematical and numerical analysis of a dynamo model

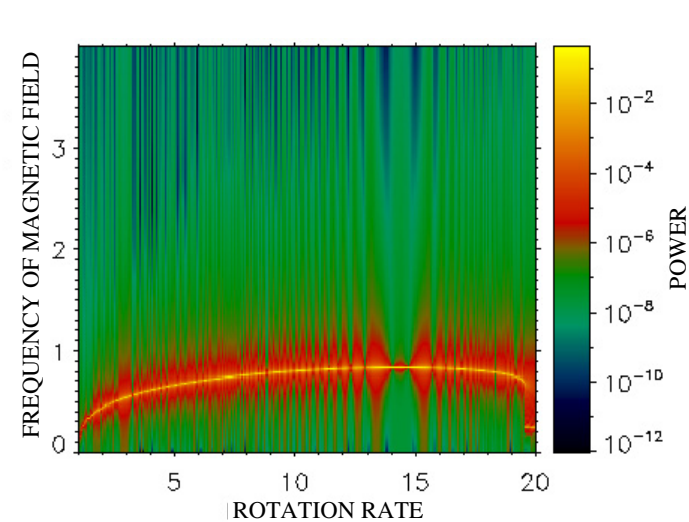

(a) $p$ as a function of rotation rate.

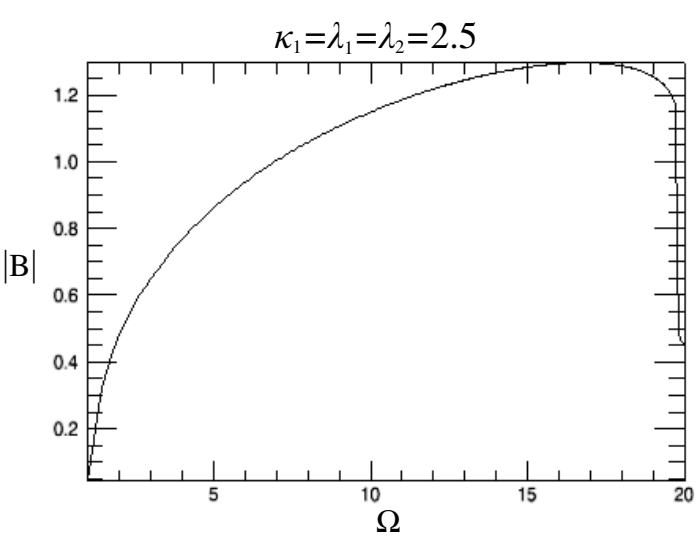

(c) $|B|$ a function of $\Omega$

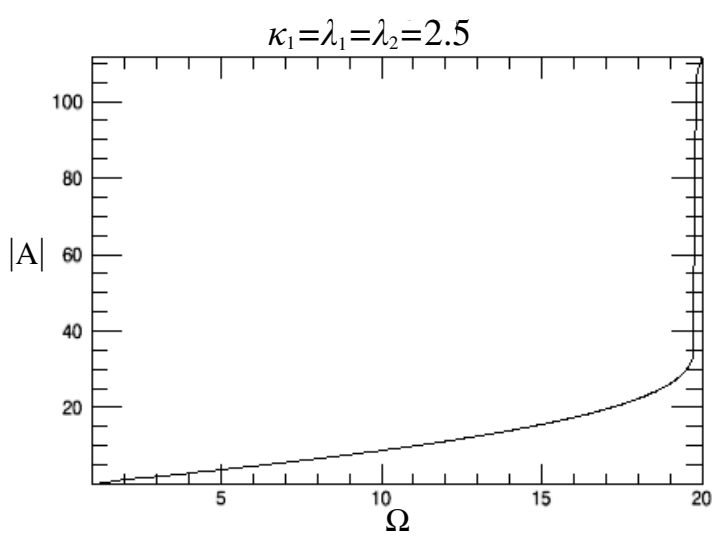

(b) $|A|$ as a function of $\Omega$

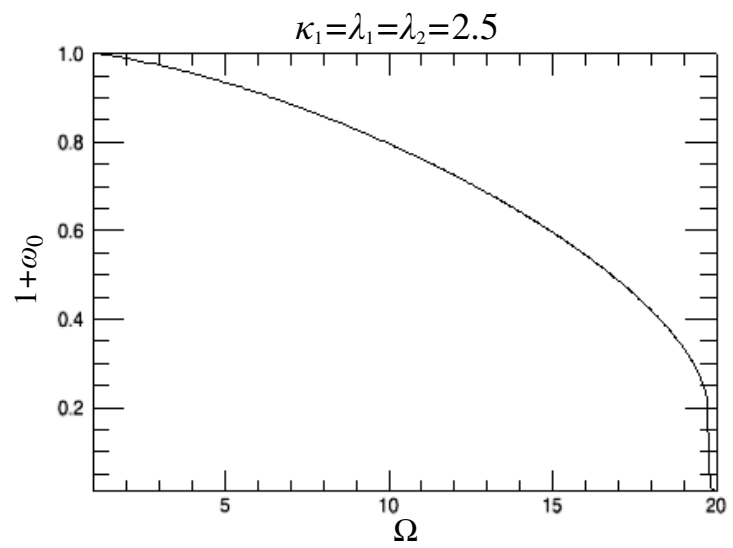

(d) Total shear as a function of rotation rate.

Fig. 5. Frequency of maximum intensity $p$, poloidal magnetic field $|A|$, toroidal magnetic field $|B|$ and total shear as a function of rotation rate $\Omega$ for $F_{1}=F_{2}=F_{3}=F$ for Case 4 in the fifth-order system.

Table 2. Scaling exponents for $p,|B|,|A|$, and $1+w_{0}$ for $\Omega \geq 5$ in the cases of the fifth-order system where $p=\Omega^{\xi},|B|=\Omega^{\beta},|A|=\Omega^{\gamma}$, and $1+w_{0}=\Omega^{\delta}$.

\begin{tabular}{lcccc}
\hline \hline Transport coefficients & $\xi$ & $\beta$ & $\gamma$ & $\delta$ \\
\hline$F_{1}=F_{2}=F_{3}=1$ & 0 & -0.98 & 1.02 & -2.0 \\
$F_{1}=F, F_{2}=F_{3}=1$ & 0 & -1.39 & 1.42 & -3.46 \\
$F_{1}=1, F_{2}=F_{3}=F$ & -0.99 & -1.56 & 1.30 & -4.30 \\
$F_{1}=F_{2}=F_{3}=F$ & varies & varies & varies & varies \\
$F_{1}=F_{2}=F, F_{3}=1$ & 0.39 & 0.45 & 0.93 & -0.16 \\
$F_{1}=F_{3}=F, F_{2}=1$ & -0.43 & -1.45 & 1.66 & -4.40 \\
$F_{1}=F_{2}=1, F_{3}=F$ & -0.30 & -1.02 & 1.20 & -2.76 \\
$F_{1}=F_{3}=1, F_{2}=F$ & -0.57 & -1.62 & 1.30 & -3.21 \\
\hline
\end{tabular}

We now compare these results with those obtained in the fourth-order system. The similarity is found in the frequency behaviour of $p$ that is controlled by the flux loss through $F_{2}$ and $F_{3}$, without being influenced by $\alpha$-quenching and also in phase shift, which is determined by ratio of $F_{2}$ and $F_{3}$. Also, it is noticed that $p$ does not change its value with rotation rate for $F_{1}=F_{2}=F_{3}=1$ and $F_{1}=F, F_{2}=F_{3}=1$, bearing a resemblance to Case 1 in Sect. 3. The similar role of $F_{2}$ and $F_{3}$ in the fourth-order system is, however, broken owing to mean differential rotation. The agreement between analytical scalings and numerical scalings becomes less accurate than the fourth-order system, with only two cases of a close match (e.g., $F_{1}=F_{2}=F_{3}=1$ and $\left.F_{1}=F_{2}=F, F_{1}=1\right)$. Both $b$ and $a$ are found to be decreased with $\Omega$ after an initial increase in all cases except $F_{1}=F_{2}=F, F_{3}=1$, which is very similar to the numerical results found for $F_{1}=F_{2}=F, F_{3}=1$ in Sect. (3). But in certain cases where the combined action of $F_{1}=F_{2}=F_{3}=F$ is considered, $A / \Omega$ grows faster than $|B|$ for higher $\Omega$.

In summary, the mean differential rotation generated by the Lorentz force severely inhibits the dynamo with significant quenching of magnetic fields for large $\Omega$. That is, without fluctuating differential rotation, quenching in mean differential rotation tends to become severe, possibly shutting down the dynamo for large $\Omega$. This thus suggests that self regulation of differential rotation through fluctuating differential rotation is needed to prevent it. This will be shown in the next section.

\section{Nonlinear effect through fluctuating differential rotation}

The goal of this section is to understand the effect of fluctuating differential rotation by assuming $w_{0}=0$ in Eqs. (6) $-(10)$. Since, analytical solutions are not particularly illuminating, Eqs. (6)-(10) are solved numerically to examine how the scalings of $a, b$, and $p$ change with $\Omega$ in different cases. We choose $v=0.5$ for these simulations to highlight the effect of nonlinear terms in taming the chaotic property of the system.

I. $F_{1}=F_{2}=F_{3}=1$ : this is the case where dynamo saturates solely due to the back reaction of fluctuating differential rotation with no nonlinear feedback through transport coefficients. 


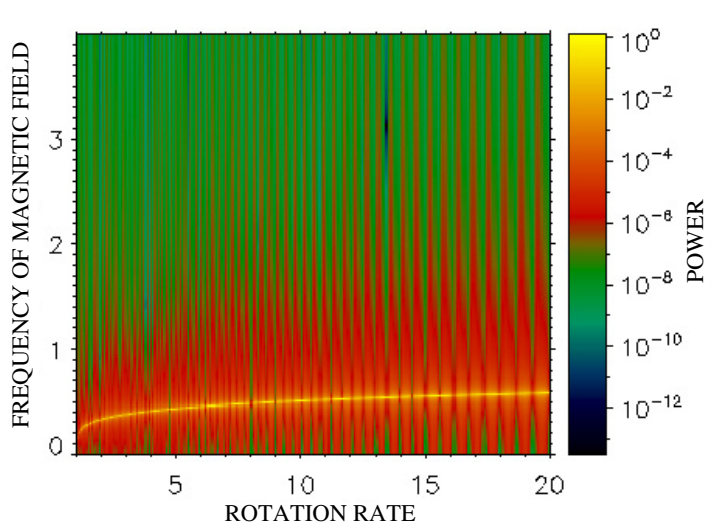

(a) $p$ as a function of rotation rate.

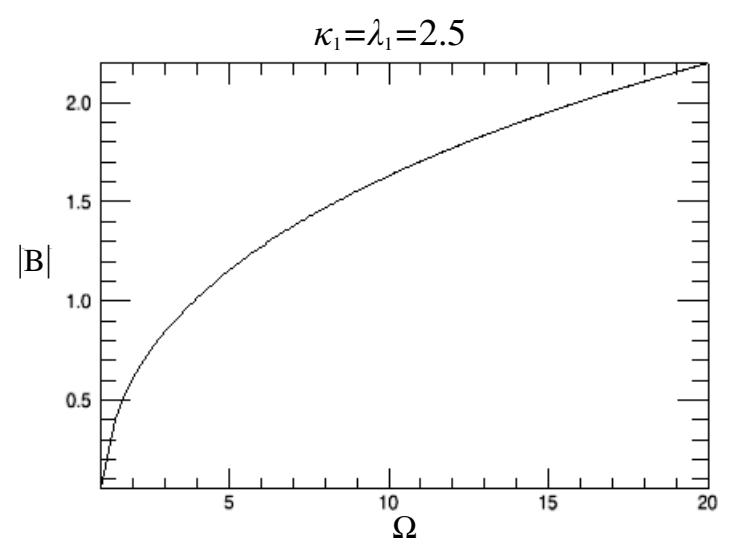

(c) $|B|$ a function of $\Omega$

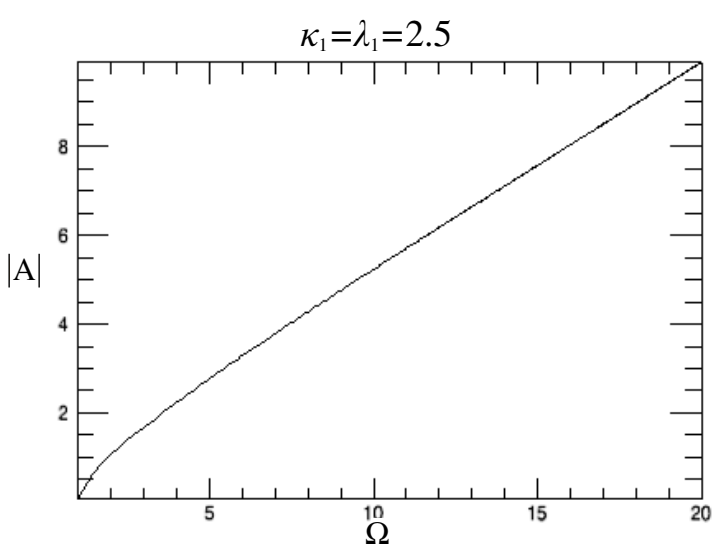

(b) $|A|$ as a function of $\Omega$

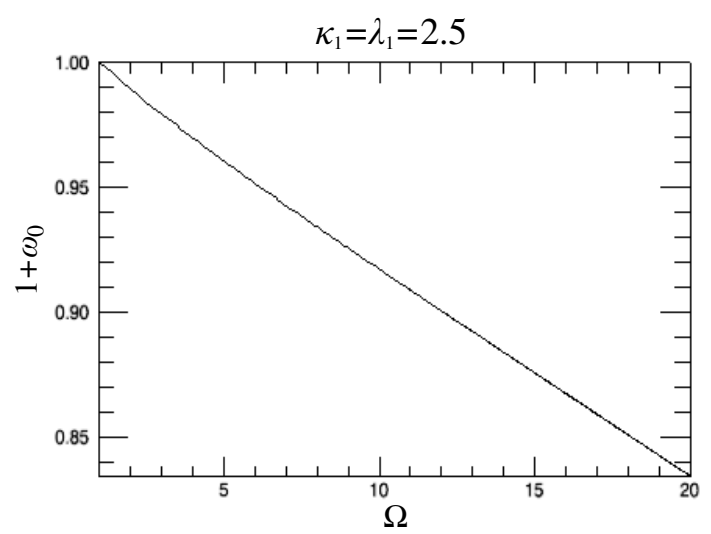

(d) Total shear as a function of rotation rate.

Fig. 6. Frequency of maximum intensity $p$, poloidal magnetic field $|A|$, toroidal magnetic field $|B|$ and total shear as a function of rotation rate $\Omega$ for $F_{1}=F_{2}=F, F_{3}=1$ for Case 5 in the fifth-order system.

Figure 7a shows the frequency spectra for different values of $\Omega$. We can see a broad band of frequency $p$ of a magnetic field of high intensity, which increases very rapidly with $\Omega$. This broad band is a manifestation of the presence of many different frequencies in the system as a result of chaotic dynamics. A localized band of lower frequencies is also noticeable, which increases with $\Omega$. The $b$ and $a$ plotted in Figs. 7b and 7c both show their rapid increase with $\Omega$ with small fluctuations appearing for large $\Omega$. Strong chaos in the system is caused by fluctuating differential rotation (cf. Figs. 7a to 7c).

II. The presence of $\alpha$-quenching or either flux loss tames the chaos, leading to a finite amplitude solution with a localized frequency. For different values of $F_{1}, F_{2}$, and $F_{3}$, the scaling results of dynamical variables for large $\Omega$ are summarized in Table 3 . The scalings $p,|B|$, and $|A|$ are highly variable with $\Omega$, especially for small $\Omega$. We thus study these as a function of $\Omega$ by computing local scalings and plot them in Figs. $8 \mathrm{a}-8 \mathrm{c}$. For different combinations of $F_{1}, F_{2}$, and $F_{3}$, we plot results using different colours/linestyles in Figs. 8a-8c. We denote the local scaling exponents of $p,|B|$, and $|A|$ as $\xi_{1}, \beta_{1}$, and $\gamma_{1}$, respectively. Clearly, the change in local scalings of $p,|B|$, and $|A|$ become less variable for high-rotation $\Omega \geq 5$ (cf. Figs. $8 \mathrm{a}-8 \mathrm{c}$ ). It is also interesting to see that the scalings of $p$ are more robust because they vary less than those in $|B|$ and $|A|$.

We first examine the effect of the inclusion of flux losses in the presence of nonlinear $\alpha$-effect. In the case of $\alpha$-quenching only (i.e., for $F_{1}=F, F_{2}=F_{3}=1$ ), $|B|$ grows rapidly with $\Omega$,
Table 3. Scaling exponents for $p,|B|$, and $|A|$ for $\Omega \geq 5$ in the cases of the sixth-order system where $p=\Omega^{\xi},|B|=\Omega^{\beta}$, and $|A|=\Omega^{\gamma}$.

\begin{tabular}{lccc}
\hline \hline Transport coefficients & $\xi$ & $\beta$ & $\gamma$ \\
\hline$F_{1}=F, F_{2}=F_{3}=1$ & 0.63 & 0.73 & 0.71 \\
$F_{1}=F_{2}=F, F_{3}=1$ & 0.57 & 0.50 & 0.78 \\
$F_{1}=F_{2}=F_{3}=F$ & 0.78 & 0.40 & 1.01 \\
$F_{1}=F_{3}=F, F_{2}=1$ & 0.81 & 0.36 & 0.95 \\
$F_{1}=1, F_{2}=F_{3}=F$ & 1.20 & 0.52 & 1.39 \\
$F_{1}=F_{2}=1, F_{3}=F$ & 1.20 & 0.44 & 1.24 \\
$F_{1}=F_{3}=1, F_{2}=F$ & - & - & - \\
\hline
\end{tabular}

whereas $p$ and $|A|$ increase very slowly with $\Omega$ (see Figs. 8a-8c). When nonlinear toroidal flux loss is added to this case (i.e., for $F_{1}=F_{3}=F, F_{2}=1$ ), $\beta_{1}$ (local scaling of magnetic field strength $|B|$ ) is decreased by approximately half, while $\xi_{1}$ (local scaling of frequency $p$ of magnetic field) and $\gamma_{1}$ (local scaling of poloidal magnetic field strength $|A|$ ) become larger. The inclusion of nonlinear poloidal flux loss to this case (i.e., for $F_{1}=F_{2}=F_{3}=F$ ) increases the $\beta_{1} \sim \beta$ by $10 \%$ with a powerlaw scaling of $\beta=0.40$ (cf. Table 3 ) in comparison with the value of $\beta_{1} \sim \beta=0.36$ in the case $F_{1}=F_{3}=F, F_{2}=1$. We note that the reduction in the value of $\xi_{1} \sim \xi$ is $4 \%$ and $\gamma_{1} \sim \gamma$ is $6 \%$. Thus, results suggest that including nonlinear poloidal flux loss in the presence of nonlinear $\alpha$ and toroidal flux loss reduces the scaling of $p$ with $\Omega$, while increases the scaling of $|B|$. This demonstrates that poloidal flux loss does not contribute much to 


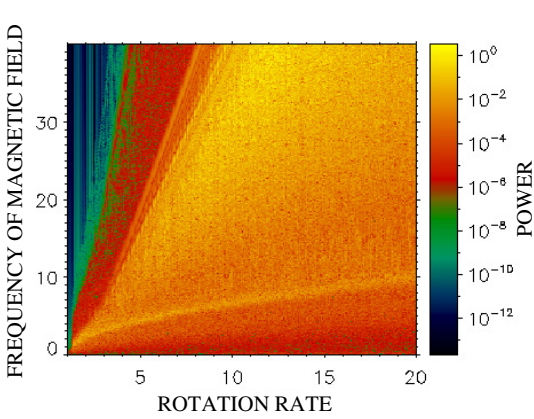

(a) $p$ as a function of $\Omega$

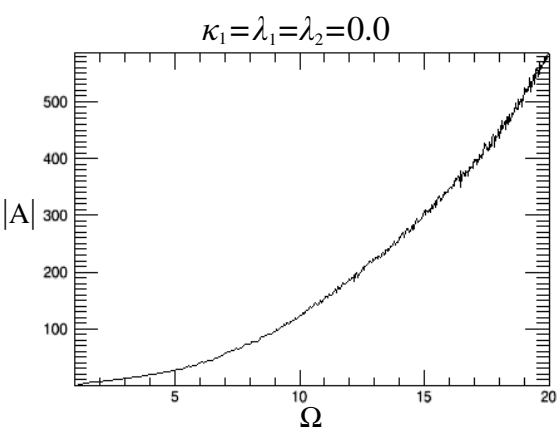

(b) $|A|$ as a function of $\Omega$

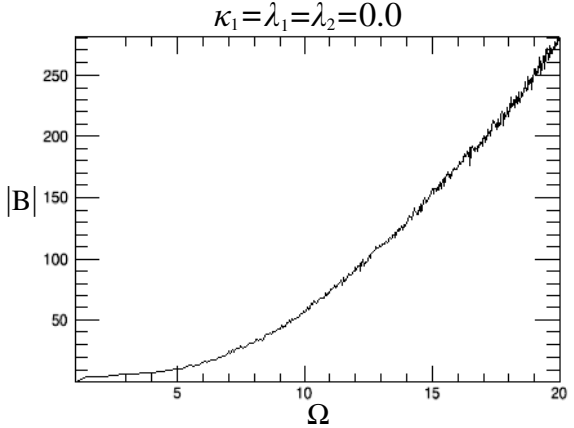

(c) $|B|$ as a function of $\Omega$

Fig. 7. Frequency of maximum intensity $p$, poloidal magnetic field $|A|$ and toroidal magnetic field $|B|$ as a function of rotation rate $\Omega$ for $F_{1}=F_{2}=$ $F_{3}=1$ for Case 1 in the sixth-order system.

the regulation of the growth of toroidal magnetic field strength with $\Omega$.

Second, we focus on the effect of flux losses when $F_{1}=1$. The equal amount of $F_{2}$ and $F_{3}$ increases the scaling of $p$, $|B|$, and $|A|$ to a value $\xi_{1} \sim \xi=1.20, \beta_{1} \sim \beta=0.52$, and $\gamma_{1} \sim \gamma=1.39$, respectively. In comparison, toroidal flux loss (i.e., for $F_{3}=F, F_{2}=F_{1}=1$ ) does not change the scaling exponent of $p$, but it is noticed to reduce the scaling of $|B|$ and $|A|$ with scaling exponents $\beta_{1} \sim \beta=0.44$ and $\gamma_{1} \sim \gamma=1.24$ in comparison with both flux losses case. Figure 8 a shows local scaling of $p$ as a function of $\Omega$ for $F_{2}=F_{3}=F$ and $F_{3}=F$. For $F_{1}=F_{3}=1, F_{2}=F, p,|b|$ and $|a|$ show fluctuation for a higher rotation rate, as can be seen from Figs. $8 \mathrm{a}-8 \mathrm{c}$, where local scalings of $p,|B|$, and $|A|$ are highly variable as $\Omega$ changes, indicating that poloidal flux loss is not efficient in reducing the effect of fluctuating differential rotation. Therefore, these results suggest that nonlinear toroidal magnetic flux loss is more important than poloidal flux loss in taming the dynamo.

In summary, the study of all cases of the sixth-order system demonstrates that the presence of fluctuating differential rotation leads to magnetic field $|B|$, which keeps increasing with $\Omega$ for high rotation with the scaling in the range $[0.36,0.73]$ (cf. Table 3). However, this increase in magnetic field is less rapid compared to the case of the fourth-order system with no fluctuating and no mean differential rotation. For instance, in the fourth-order system, the scaling of magnetic field $|B|$ with $\Omega$ are $[0.41,1.0]$ (cf. Table 1). Toroidal flux loss is more important in reducing $\beta_{1}$ as a nonlinear term than poloidal flux loss. These results show that in the sixth-order system various saturation mechanisms, especially, $\alpha$-quenching and toroidal flux loss, are able to slow down the growth of magnetic field with $\Omega$ to some degree, but not strongly enough to flatten the magnetic field for large $\Omega$. Compared to the results in the fourth-order system, the opposite effects of mean differential rotation and fluctuating differential rotation shown in Sects. 4 and 5, respectively, suggest that the combined effect of both mean and fluctuating differential rotation is beneficial for the self-regulatory behaviour of dynamo. These opposite effects of mean and fluctuating differential rotation also make the effects of toroidal and poloidal flux loss equally important in the seventh-order system, as discussed in Sect. 6.

\section{Implications and key observations}

Ever more improved observational data of the evolution of magnetic fields and rotations of various stars of different age and spectral types with different rotation rates have been revealing valuable information about the relation among rotation, differential rotation, and magnetic activity. First, the cycle period $P_{\text {cyc }}$ of magnetic fields of the solar type stars is shown to depend upon the stellar rotation period $P_{\text {rot }}$ as $P_{\text {cyc }} \propto P_{\text {rot }}^{\beta}$, with scaling exponent $\beta=1.25 \pm 0.5$ (Noyes et al. 1984); e.g., $\beta=0.8$ for the active branch and $\beta=1.15$ for the inactive branch (Saar \& Brandenburg 1999; Charbonneau \& Saar 2001; Saar 2002). Second, the magnetic activity (a measure of the strength of magnetic fields) has been observed to increase with stellar rotation but tends to saturate for high rotation (with $P_{\text {rot }}<3$ day). Third, the differential rotation $\Delta \Omega$ is observed to increase with the stellar rotation rate $\Omega$, while the relative difference $\Delta \Omega / \Omega$ decreases with $\Omega$. The precise dependence of differential rotation on rotation is quite uncertain since $\Delta \Omega \propto \Omega^{n}$ where $0<n<1$ (Fröhlich et al. 2009; Hotta \& Yokoyama 2011; Donahue et al. 1996; Reiners \& Schmitt 2003; Barnes et al. 2005). Furthermore, the time variation of differential rotation has been reported in recent work (e.g., Fröhlich et al. 2012; Hotta \& Yokoyama 2011).

Therefore, a main challenge in dynamo theory is to explain these observations. For instance, the almost linear relation between the frequency of magnetic fields and stellar rotation could be explained within the framework of linear dynamo theory, for instance, by the linear dispersion relation in $\alpha-\Omega$ dynamo. However, observed magnetic fields are manifestations of nonlinear saturation, making the understanding of how stellar magnetic fields apparently manage to almost maintain a linear dependence of their frequency on rotation rate as one of the fundamental questions in any dynamo theory.

Our results from Sects. 3-5 above show that none of the cases considered are consistent with all these observations. Towards understanding these observations, Sood \& Kim (2013) have numerically studied the seventh-order system, given by Eqs. (1)-(4), and performed a thorough parameter study on the choice of nonlinear quenching effects of $F_{1}, F_{2}$, and $F_{3}$ by using $v=1$ and $v_{0}=35$. From their investigation, they proposed a minimal model that can reproduce the observed relation between magnetic frequency and rotation rate. In particular, they highlight the importance of the regulatory behaviour of dynamo through a nonlinear balance between the generation and destruction of the magnetic fields. They also demonstrate the importance of both mean and fluctuating differential rotations in achieving dynamical balance among magnetic fields. We now show how, through the balance among different nonlinear effects of transport coefficients and mean and fluctuating differential rotations, this regulatory behaviour arises in the seventh-order 


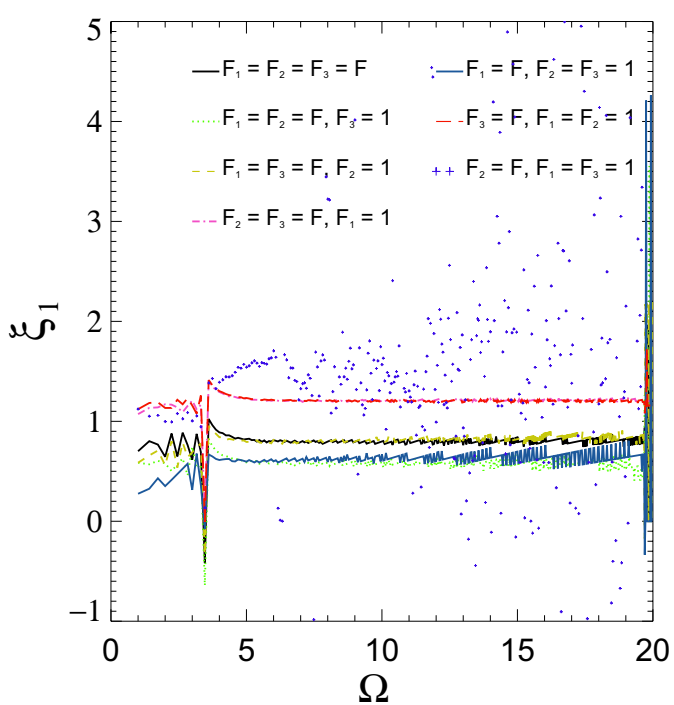

(a) $\xi_{1}$ as a function of $\Omega$

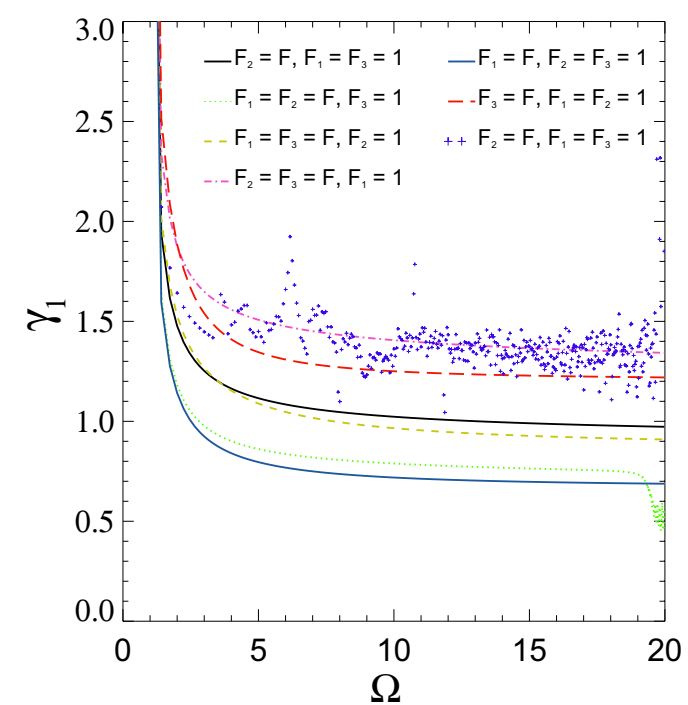

(b) $\gamma_{1}$ as a function of $\Omega$

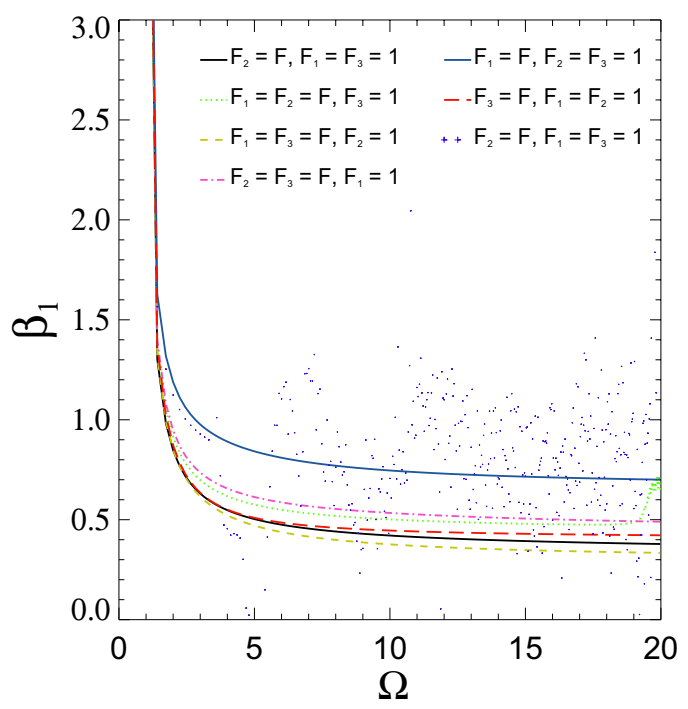

(c) $\beta_{1}$ as a function of $\Omega$

Fig. 8. Local scalings $\xi_{1}$ for $p, \gamma_{1}$ for $|A|$, and $\beta_{1}$ for $|B|$ are plotted as functions of rotation rate for different combinations of $F_{1}, F_{2}$, and $F_{3}$, where for different combinations, different colours/linestyles are assigned as: for $F_{1}=F_{2}=F_{3}=F$ black/solid line, $F_{1}=F_{2}=F, F_{3}=1$ green/dot, $F_{1}=F_{3}=F, F_{2}=1$ gold $/$ dash, $F_{1}=1, F_{2}=F, F_{3}=F$ pink $/$ dash dot, $F_{1}=F, F_{2}=F_{3}=1$ turquoise $/$ dash dot dot, $F_{1}=F_{2}=1, F_{3}=F$ red $/$ long dash, $F_{1}=F_{3}=1, F_{2}=F$ turquoise/“+".

system when $F_{1} \sim F_{2} \sim F_{3}$. To demonstrate how the results in Sects. 4-6 are altered in this seventh-order system, we use the same parameter values $v=0.5$ and $v_{0}=35$ used in Sects. 4-6.

The behaviour of frequency of magnetic field and magnetic field strength with rotation rate in this seventh-order system is shown in Figs. 9a-9b. Figure 9a shows an almost linear increase in the frequency $p$ of maximum intensity (depicted in yellow) with rotation rate. A red band of the localized frequency of maximum intensity around $p$ is found to gradually increase its width with rotation rate. The power-law dependence of $p$ on $\Omega$ is found to be $p \sim \Omega^{0.82}$ for a higher rotation rate and agrees with observations. The flattening of magnetic field strength $|B|$ for a higher rotation rate can be seen in Fig. 9b, which is also consistent with observations.

To investigate the balance, in Figs. $10 \mathrm{a}-10 \mathrm{~b}$, we plot the magnitude of various nonlinear terms appearing in Eqs. (1), (2) for $D_{0}=2$, which are $|2 D B|,\left|F_{2} A\right|,\left|\mathrm{i}\left(1+w_{0} \mid\right) A,\right| F_{3} B \mid$, and $\left|\frac{1}{2} \mathrm{i} A^{*} w\right|$. Figure 10a shows that there is a balance between the $\alpha$-quenching term $|2 D B|$ and poloidal flux loss term $\left|F_{2} A\right|$, depicted in black and red, respectively. This thus indicates that the dissipation and the generation of magnetic field are almost balanced in Eq. (1). Figure 10b shows that the generation of poloidal field due to shear $\left|w_{0} A\right|$ (illustrated in black) is in balance with toroidal flux loss $\left|F_{3} B\right|$ (represented in red). Fluctuating differential rotation $\left|\frac{1}{2} \mathrm{i} A^{*} w\right|$ depicted in blue is less dominant in this case. Thus, the two main nonlinear terms in Eq. (2) are of similar magnitude.We compare the nonlinear terms in Eqs. (1) and (2) for all other cases in seventh-order systems and confirm that the dynamical balance is best achieved for this seventh-order system with $F_{1}=F_{2}=F_{3}$.

\section{Conclusions}

We studied nonlinear dynamical model analytically and numerically to elucidate the effects of nonlinear terms in the regulation of a dynamo. In the fourth-order system, flux losses 
A. Sood and E. Kim: Detailed mathematical and numerical analysis of a dynamo model

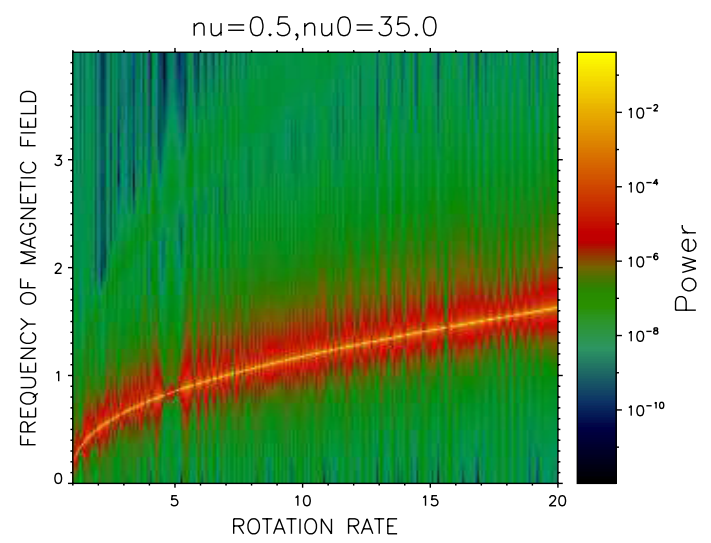

(a) $p$ as a function of rotation rate $\Omega$.

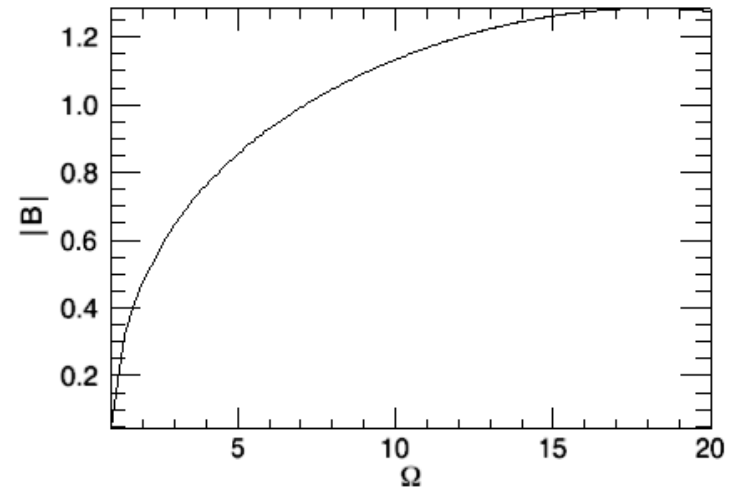

(b) Magnetic field strength $|B|$ as a function of rotation rate $\Omega$.

Fig. 9. Frequency $p$ of magnetic field and toroidal magnetic field $|B|$ are plotted as a function of rotation rate $\Omega$ for $F_{1}=F_{2}=F_{3}=F$ in case of the seventh-order system for $v=0.5$ and $v_{0}=35.0$.

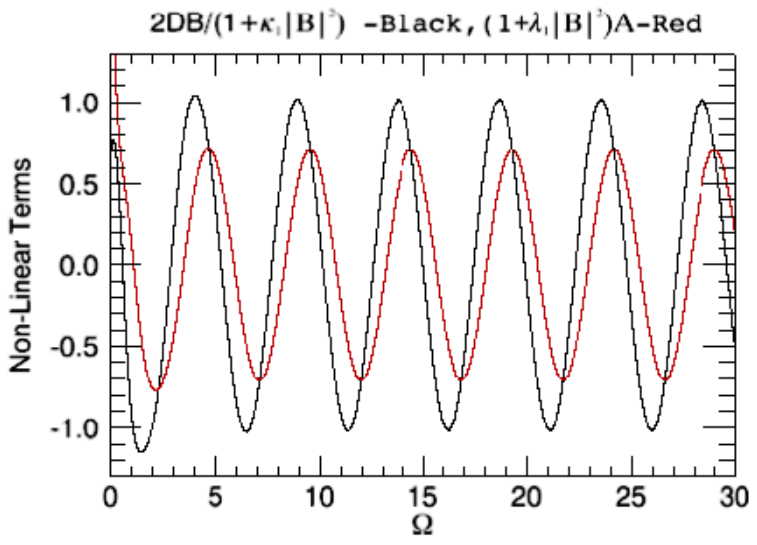

(a) Dynamical balance between nonlinear terms on R.H.S. of Eq. (1). $\left(1+w_{0}\right) A-B l a c k,\left(1+\lambda_{2}|B|^{2}\right) B-$ Red, $-0.5 i A * w-B l u e$

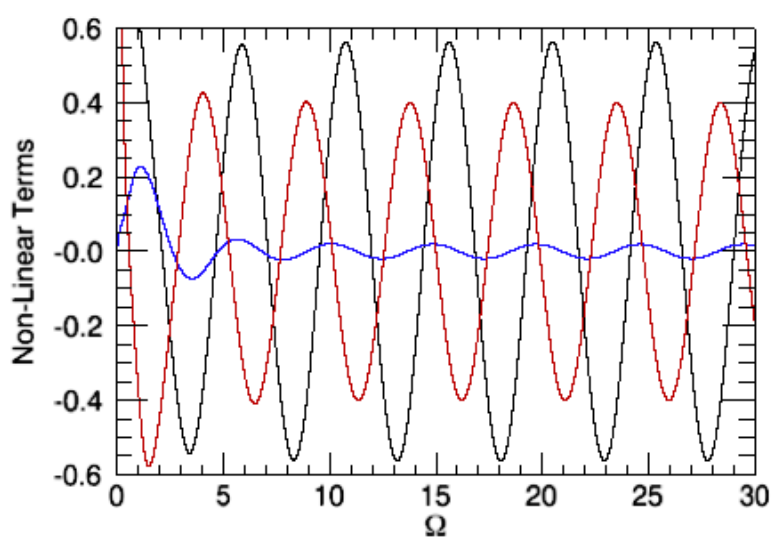

(b) Dynamical balance between nonlinear terms on R.H.S. of Eq. (2).

Fig. 10. Time series of different nonlinear terms for $F_{1}=F_{2}=F_{3}=F$ in case of the seventh-order system for $v=0.5$ and $v_{0}=35.0$.

determine the frequency $p$ of magnetic field, without any influence of $\alpha$-quenching; that is, $\alpha$-quenching does not affect the frequency $p$. The effect of flux losses due to poloidal magnetic field and toroidal magnetic field is the same on $p$ and $|B|$ irrespective of the presence or absence of $\alpha$-quenching. Phase $\phi$ is determined by the ratio of $F_{2}$ and $F_{3}$. We observed the increase in $p$ and $|B|$ with $\Omega$ in all cases, with power-law scalings $\xi \sim[0,1.0]$ and $\beta \sim[0.4,1.0]$, respectively. We find good agreement between analytical and numerical results in this system.

The presence of shear due to mean differential rotation, that is, in the fifth-order system, frequency $p$ keeps a constant value $p=1$ for different rotations when the $\alpha$-effect is quenched or when there are no transport coefficients present in the system. That is, again the frequency $p$ is determined by both flux losses and phase is determined by the ratio of $F_{2}$ and $F_{3}$, which is similar to the fourth-order system. But the effects of $F_{2}$ and $F_{3}$, with or without $\alpha$-quenching, are no longer the same on $p$ and $|B|$ in contrast to the fourth-order system. We find growth of $p$ and $|B|$ in the presence of an equal combination of $\alpha$-effect and poloidal flux loss (i.e., $F_{1}=F_{2}=F, F_{3}=1$ ). The quenching of magnetic field $|B|$ and $p$ is more significant when the nonlinear $\alpha$-effect and toroidal flux loss are taken together (i.e., $F_{1}=F_{3}=F, F_{2}=1$ ). The decreasing behaviour of $p$ and $|B|$ in almost all cases is due to severe shear quenching, which is responsible for the shutting down of dynamo action.
In the sixth-order system, we present local scalings with rotation rate to demonstrate the effect of nonlinear terms. Here, unlike the fourth- and the fifth-order systems, $\alpha$-quenching (i.e., $F_{1}$ ) influences $p$, while $p$ and $|B|$ behave differently when the effect of either flux loss is considered in the presence or absence of $\alpha$-quenching. Interestingly, in this case, $p$ and $|B|$ always increase with $\Omega$ despite the feedback from fluctuating differential rotation. Toroidal flux loss is shown to be more important than poloidal flux loss. The results observed for the fifth-order and the sixth-order systems thus identify the pivotal role of the combined effect of both mean and fluctuating differential rotation in the self regulation of a dynamo to work near marginal stability. We also note that the self regulation between generation and dissipation of magnetic fields is a prerequisite for working of the dynamo. In all cases, the scaling of $p$ is less sensitive to the change in $\Omega$ or to the different combinations of nonlinear transport coefficients.

Finally, we presented how this self-regulatory behaviour comes about in the seventh-order system in the presence of an equal combination of $\alpha$-quenching, poloidal flux loss, and toroidal flux loss (Sood \& Kim 2013), where $\alpha$-quenching, toroidal flux loss and poloidal flux loss are equally important. While our work is based on the $\alpha-\Omega$ dynamo model, similar regulation of the generation and the dissipation of magnetic fields not only between nonlinear transport coefficients but also between mean and fluctuating differential rotation is likely to 
persist in other models. The extension of this work to other models will be addressed in a future paper.

It will also be of great interest to utilize our model to understand the spin down of solar type stars (Leprovost \& Kim 2010; Kepens et al. 1995) by using a magnetic field that is consistently obtained from a dynamo model (work in progress).

\section{References}

Barnes, J. R., Collier Cameron, A., Donati, J.-F., et al. 2005, MNRAS, 357, L1

Charbonneau, P. 2005, Liv. Rev. Sol. Phys., 2, 2

Charbonneau, P., \& Saar, S. H. 2001, in Magnetic Fields across the H-R digram, eds. G. Mathys, S. K. Solanki, \& D. T. Wickamasinghe, ASP Conf. Ser., 248, 189

Dikpati, M., \& Charbonneau, P. 1999, ApJ., 518, 508

Donahue, R.A., Saar, S. H., \& Baliunas, S. L. 1996, ApJ, 466, 384

Fröhlich, H.-E., \& Arlt, R. 2012, A\&A, 543, A7

Fröhlich, H.-E., Küker, M., Hatzes, A. P., \& Strassmeier, K. G. 2009, A\&A, 506, 263

Hotta, H., \& Yokoyama, T. 2011, ApJ. 740, 1

Ivanova, T. S., \& Ruzmaikin, A. A. 1977, Sov. Astron., 21, 479

Jepps, S. A. 1975, J. Fluid Mech., 67, 625

Jones, C. A., Weiss, N. O., \& Cattaneo, F. 1985, Physica D, 14, 161

Kepens, R., MacGregor, K. B., \& Charbonneau, P. 1995, A\&A, 294, 469

Kim, E., \& Diamond, P. H. 2003, Phys. Rev. Lett., 90, 185006

Kitchatinov, L.L., Rüdiger, G., \& Kükker, M. 1994, A\&A, 292, 125

Kleeorin, N. I., \& Ruzmaikin A. A. 1982, Magnetohydrodynamics, 18, 116

Krause, F., \& Rädler, G. 1980, Mean-Field Magnetohydrodynamics and Dynamo Theory (Oxford: Pergamon Press)

Leprovost, N., \& Kim, E. 2010, ApJ., 719, 287
Lopes, I., \& Passos, D. 2009, MNRAS, 397, 320

Lopes, I., \& Passos, D. 2011, J. Atmos. Sol. Terr. Phys., 73, 191

Malkov, M. A., \& Diamond, P. H. 2009, Phys. Plasmas, 16, 012504

Malkus, W. V. R., \& Proctor, M. R. E. 1975, J. Fluid Mech., 67, 417

Mininni, P. D., Gomez, D. O., \& Mindlin, G. B. 2001, Sol. Phys., 201, 203

Moffatt, H. K. 1978, Magnetic Field Generation in Electrically Conducting Fluids (Cambridge: Cambridge University Press)

Noyes, R.W., Weiss, N. O., \& Vaughan, A. H. 1984, ApJ, 287, 769

Parker, E. N. 1955, ApJ, 122, 293

Parker, E. N. 1979, Cosmical Magnetic Fields (Oxford: Clarendon Press)

Passos, D., \& Lopes, I. 2008, ApJ, 686, 1420

Pontieri, A., Lepreti, F., Sorriso-Valvo, L., Vecchino, A., \& Carbone, V. 2003, Sol. Phys., 213, 195

Pouquet, A., Frisch, U., \& Leorat, J. 1976, J. Fluid Mech., 77, 321

Reiners, A., \& Schmidt, J. 2003, A\&A, 398, 647

Rempel, M. 2006, ApJ, 647, 662

Saar, S. H. 2002, in the 11th Cool Stars: Stellar System and the Sun, eds. R. J. Garcia Lopez, R. Rebolo, \& M. R. Zapatero, ASP Conf. Ser., 223, 292

Saar, S. H., \& Brandenburg, A. 1999, ApJ, 524, 295

Sood, A., \& Kim, E. 2013, A\&A, 555, A22

Tobias, S. M., Weiss, N. O., \& Kirk, V. 1995, MNRAS, 273, 1150

Weiss, N. O., Cattaneo, F., \& Jones, C. A. 1984, Geophys. Astrophys. Fluid Dynamics, 30, 305

Weiss, N. O., Knobloch, E., \& Tobias, S. M. 2001, Nato Science Series 26 (Springer Verlag), 381

Wilmot-Smith, A. L., Martens, P. C. H., Nandy, D., Presit, E. R., \& Tobias, S. M. 2005, MNRAS, 363, 1167

Yoshimura, H. 1975, ApJS, 29, 467

Yoshimura, H. 1978, ApJ, 220, 692

Zhu, H., Chapman, S. C., \& Dendy, R. O. 2013, Phys. Plasmas, 20, 042302 